\title{
Hick's law: Its limit is 3 bits
}

\author{
LANGDON E. LONGSTRETH \\ University of Southern California, Los Angeles, California
}

\begin{abstract}
Hick's law states that choice reaction time is a linear function of the amount of information provided by the reaction stimulus. Thus a straight line is predicted for the function that relates choice reaction time to the logarithm of the number of response alternatives. Although some (but not all) studies have confirmed this relationship, the range of response alternatives has been almost completely limited to 2 to 8 choices. Three experiments now extend the range to 16 choices and beyond. In all of them, a curvilinear function is obtained. Longstreth's power law better fits the data (Longstreth, El-Zahhar, \& Alcorn, 1985). It is concluded that Hick's law is an approximation to the complete power function and that it becomes noticeably deficient only beyond 3 bits of stimulus uncertainty (i.e., 8 choices).
\end{abstract}

In 1952, W. E. Hick, a colleague of A. T. Welford, reported a remarkably simple relationship between choice reaction time (CRT) and number of equiprobable and independent response alternatives $(n)$ (Hick, 1952):

$$
\mathrm{CRT}=K \log (n+1) .
$$

This linear relationship has been confirmed many times; however, it is not without its problems. One criticism is that there are exceptions to the law. As it turns out, certain kinds of tasks produce a flat reaction-time function across bits of stimulus uncertainty. The nature of these tasks has been summarized elsewhere (Longstreth, ElZahhar, \& Alcorn, 1985).

A second criticism is more serious, to the effect that the law not only has exceptions but that it is, in fact, merely an artifact of a restricted range of stimulus uncertainty. Almost all studies of Hick's law have restricted the number of choices to a maximum of 8 , with the number of choices varied among 2,4 , and 8 . Three points, of course, is the minimum number required to detect a nonlinear function. This criticism was first suggested in 1985 (Longstreth et al., 1985), and has since been challenged by Welford (1987). A necessarily brief rebuttal was offered (Longstreth \& Alcorn, 1987). I now expand that rebuttal by reporting the results of three experiments in which number of choices extends beyond 8 .

\section{EXPERIMENT 1}

Experiment 1 explored the CRT function between 1 bit ( 2 choices) and 4.4 bits ( 21 choices). Set sizes of 2,3 , $6,10,15$, and 21 were employed. This variation was accomplished with six response keys and six digits by including all pairs of digits as stimuli as well as all single digits. Thus, for set size 3 , the possible stimuli were 4 ,

\footnotetext{
The author thanks Jia Pan for assistance in running subjects. Correspondence should be addressed to Langdon E. Longstreth, Psychology Department, University of Southern California, Los Angeles, CA 90089.
}

7, and 47. The correct response for a double digit was to press both keys simultaneously. (Only responses to single digits constituted the data to be analyzed, as subsequently described.) For the largest set size, the digits $2,3,4,7,8$, and 9 were employed, and all combinations of two digits (15), for a total of 21 possibilities.

\section{Method}

Subjects and Apparatus. Thirteen undergraduate developmental psychology students served as subjects. The entire procedure was presented and controlled via an Apple II microcomputer and monitor. The subject sat facing the monitor with his/her hands on the keyboard. Location of the digits on the monitor was physically isomorphic with the associated keyboard response keys. Each digit was displayed directly above its corresponding key, $\pm 0.5 \mathrm{~cm}$. Thus the stimulus pattern consisted of one to three digits on each side of the screen, with a gap between the two groupings corresponding to the keyboard gap between 4 and 7 . Stimuli and responses were therefore extremely " compatible," to use the terminology of Fitts and Switzer (1962).

The fourth, third, and second fingers of the left hand actuated keys 2,3 , and 4 , and the second, third, and fourth fingers of the right hand actuated keys 7,8 , and 9 . The subject was instructed to place his/her fingers on the keys that would be utilized at each set size. The remaining keys were to remain uncovered to the extent that it was comfortable to do so. Most subjects placed a curled finger on the relevant keys (e.g., keys 4 and 7 for set sizes 2 or 3), and a flat finger near or on the irrelevant keys.

Procedure. The subjects were first informed of the purpose of the task, that is, to measure reaction time, and were then shown a few demonstration trials. Then each subject received a block of 10 practice trials at each set size. Block order was the same as would be used in the task proper, and was randomly determined for each subject. Following practice, the subject was exposed to 20 trials at each set size. At the beginning of each block of trials, the subject was advised of the set size and appropriate response keys for that block. At the end of each block (about 3-4 min later), a 2-min rest period ensued while a printout of reaction times was obtained and the program was reset for the next block of trials.

The reaction stimulus on each trial was determined randomly with the following restriction: at set sizes 10,15 , and 21, " 4 ," " 7 ,", and " 47 " constituted the reaction stimulus on Trials 16,18 , and 20 , respectively. The goal of this restriction was to ensure that these reaction stimuli appeared at least once at each set size greater than 2 , and that " 4 " and " 7 " appeared at least once at all set sizes. Reaction time of correct responses to these two common stimuli constituted the dependent variable. 


\section{Results}

Preliminary analyses showed that number of errors in this task was acceptably small, less than $5 \%$ at all set sizes. Median reaction time to the combined common stimuli at each set size (4 and 7) was determined for each subject. The means of these medians are presented as the solid curve in Figure 1. This function is negatively accelerated, with significant linear and quadratic components $(p<$ .01). I will delay further comments, but for now it may be noted that the function from 1 to 3.32 bits is approximately linear, in keeping with Hick's law. It is only at the two larger set sizes that curvilinearity becomes obvious.

\section{EXPERIMENT 2}

The main variation in Experiment 2 was a mixed order of set sizes rather than a blocked order. Each cycle of four trials consisted of set sizes $2,8,4$, and 16 , in that order, with four repetitions of this cycle in each of six blocks of trials. Another variation was to restructure the physical compatibility of the task in such a way as to increase its similarity to that of a currently popular procedure involving only one finger. Jensen (1983) reported a large number of studies of his own and by others, all of which use the same task. In this task, the subject presses a home button until one of eight spatially distinct lights is activated. The task is to remove the finger from the home button and press a button under the activated light as quickly as possible. With this task, Jensen has repeatedly found a linear function relating bits of uncertainty and reaction time between 1 and 3 bits. The question addressed here is whether or not a linear function holds at greater bit values (specifically, from 1 to 4 bits).

\section{Method}

Apparatus and Procedure. The stimuli in this task were the numbers 1 through 16. The subject's task was to depress the home button (space bar) to receive the reaction stimulus, and then to hit the corresponding key as rapidly as possible without making an error. To this end, white tabs were pasted on keys 1 through 0 , identifying them as " 1 " through " 10 ," and six tabs were pasted on the left-most second row (Keys Q, W, E, R, T, and Y) identifying them as "11" through
"16." Reaction time was defined as the temporal interval between depression of the space bar (stimulus onset) and depression of one of the 16 response keys, both responses being executed by the same hand and finger.

At set size 2, the stimuli were the numbers 5 and 6 ; at set size 4 , 4 through 7 ; at set size 8,2 through 9 ; and at set size 16,1 through 16. As a result, 5 and 6 were common at all set sizes.

Six blocks of trials were administered, each block consisting of four runs through the set-size sequence of $2,8,4$, and 16 choices. The subjects were 10 undergraduate students earning course credit in an introductory psychology course.

\section{Results}

Means of median reaction times to common stimuli are presented in Figure 1, with the curve labeled "Exp. 2."' These means are about $200 \mathrm{msec}$ greater than those of "Exp. 1," due to the greater complexity of the response. Their values are read off the left ordinate, whereas the values for the first experiment are read off the right ordinate. The curve is again negatively accelerated. Putting the results of these two experiments together, I conclude that the curvilinear functions are not an artifact of a procedure specific to one or the other of the studies.

\section{EXPERIMENT 3}

Although Experiment 2 confirmed the curvilinear reaction-time function, it could be argued that the procedure was questionable. The trial-by-trial variation in set size may have been conducive to ignoring set size altogether, since the presumed response preparation had to be changed on every trial (e.g., Welford, 1980). One way to detect such a tendency is to compare slope of the reaction-time gradient: the smaller the slope, the less the tendency to attend to set size. In the present case, the overall slope of Experiment 2 is about half that of Experiment 1 (see Figure 1). In view of this discrepancy, Experiment 3 reverted to a block presentation of each set size.

\section{Method}

Procedure. The task was the same as in Experiment 2, except that set size was blocked as in Experiment 1. In addition, the home button was moved from the space bar to the G key located near the center of

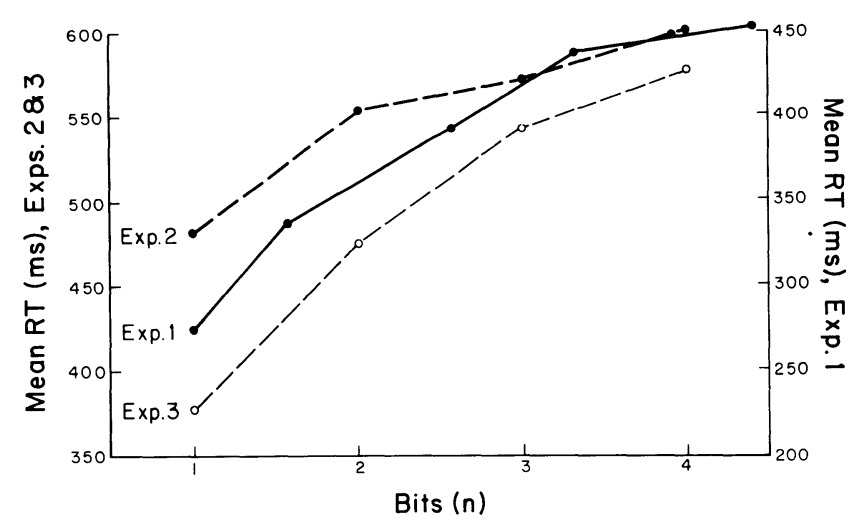

Figure 1. Mean reaction time (RT) as a function of stimulus uncertainty (bits). 
the keyboard. The reason for this modification was to minimize the distance between the home button and the response keys for the common stimuli, from about $7.5 \mathrm{~cm}$ to $3 \mathrm{~cm}$. The greater this distance, the greater the reaction time. The greater the reaction time, the greater the possibility that the response decision may not be completed at the instant of home-button release, since the additional movement time may allow further processing. In such a case, reaction time underestimates decision time. Welford (1986) recently criticized Jensen (1983) in this respect, because Jensen used an apparatus that involved a distance of about $15 \mathrm{~cm}$

Twelve practice trials each were administered at set sizes $2,4,8$, and 16 , in either an ascending order $(n=10)$ or a descending order $(n=10)$. This cycle was then repeated for the test trials, in the same set-size order. The events defining a trial were the same as in Experiment 2.

\section{Results}

The curve labeled "Exp. 3" in Figure 1 reveals another negatively accelerated function. Note again that the fit is quite linear up to 3 bits $(r=0.992)$. At 4 bits $(r=.971)$, it is still a good fit, but is nevertheless a curvilinear function.

\section{GENERAL DISCUSSION}

These three experiments provide evidence that Hick's law applies to only a restricted range of stimulus uncertainty, up to 3 bits at best. Two of the curves in Figure 1, and many others summarized elsewhere, show curvilinearity even within 3 bits (see Longstreth et al., 1985). Longstreth et al. derived a power law from a simple theory of CRT that fits these exceptions quite well.

Other studies using a range substantially beyond 3 bits are rare. Welford alludes to only one such study in his critique of the power law (Welford, 1987). This study, by Hilgendorf (1966), used up to 1,000 choices. Welford reported that a linear fit was much closer than a power fit, with amount of variance accounted for equaling 0.976 and 0.533 , respectively. Longstreth and Alcorn (1987) discounted this study because of the unique definition of response latency: the time between release of a home button and its subsequent depression, with the choice response sandwiched between these two events. We are aware of two other studies (Siebel, 1962, 1963). The maximum numbers of choices in these studies were 31 and 1,023, respectively. The functions in both studies are decidedly curvilinear. Therefore, restricting the sample of studies to these two studies plus that of Hilgendorf, plus the three present studies, all using a range extending beyond 10 choices, five of six report a curvilinear function.

\section{REFERENCES}

FitTs, P. M., \& SwITZER, G. (1962). Cognitive aspects of information processing: I. The familiarity of S-R sets and subsets. Journal of Experimental Psychology, 63, 321-329.

HICK, W. E. (1952). On the rate of gain of iniormation. Quarterly Journal of Experimental Psychology, 4, 11-26.

HiLgENDORF, L. (1966). Information input and response time. Ergonomics, 9, 31-37.

Jensen, A. R. (1983). Methodological and statistical techniques for the chronometric study of mental abilities. In C. Reynolds \& V. Willson (Eds.), Methodological and statistical advances in the study of individual differences (pp. 51-116). New York: Plenum.

LONGSTRETH, L. E., \& AlCoRN, M. B. (1987). Hick's law versus a power law: Reply to Welford. Journal of Experimental Psychology: General, 116, 315-316.

Longstreth, L. E., El-ZahHar, N., \& Alcorn, M. B. (1985). Exceptions to Hick's law: Explorations with a response duration measure. Journal of Experimental Psychology: General, 114, 417-434.

SEIBEL, R. (1962). Discrimination reaction time as a function of the number of stimulus-response pairs and the self-pacing adjustment of the subject. Psychological Monographs, 76(Whole No. 561).

SEIBEL, R. (1963). Discrimination reaction time for a 1,023-alternative task. Journal of Experimental Psychology, 66, 215-226.

WeLFord, A. T. (1980). The single-channel hypothesis. In A. T. Welford (Ed.), Reaction times. New York: Academic Press.

Welford, A. T. (1987). Comment on "Exceptions to Hick's law: Explorations with a response duration measure" (Longstreth, El-Zahhar, \& Alcorn, 1985). Journal of Experimental Psychology: General, 116, 312-314.

(Manuscript received for publication July 6, 1987.) 